\title{
Prediction of Impact Shock Vibrations at Tennis Player's Wrist Joint: Comparison between Conventional Weight Racket and Light Weight Racket with Super Large Head Size*
}

\author{
Yoshihiko KAWAZOE*2, Yukihiro TAKEDA*3 ${ }^{*}$, Masamichi NAKAGAWA*2, \\ Federico CASOLO ${ }^{*}$, Ryoso TOMOSUE* ${ }^{5}$ and Keiko YOSHINARI*6 \\ *2Department of Human-Robotics, Saitama Institute of Technology, \\ 1690, Fukaya, Saitama, 369-0293, Japan \\ E-mail: kawazoe@sit.ac.jp \\ ${ }^{*}$ Graduate School of Engineering, Saitama Institute of Technology \\ ${ }^{*}$ Politecnico di Milano, Piazza Leonardo da Vinci 32, 20133 Milano, Italy \\ *5asuda Women's College,6-13-1, ando,Asaminami, Hiroshima, 731-0153,Japan \\ ${ }^{* 6}$ Shirayuri College,1-25,Midorigaoka, Chofu, Tokyo, 182-8525, Japan
}

\begin{abstract}
The lightweight racket with handle-light configuration and large head size is recent tendency of high-tech tennis rackets, increasing power or post-impact ball velocity with an increasing racket swing speed. This paper investigated the performance of lightweight tennis racket with super-large head size in terms of feel or comfort. It predicted the effect of the mass and mass distribution of super-large sized rackets on the impact shock vibrations of the racket handle and the player's wrist joint when a player hits a flat forehand drive. The prediction is based on the identification of the racket characteristics, the damping of the racket-arm system, equivalent mass of the player's arm system and the approximate nonlinear impact analysis in tennis. A super-light weight balanced racket (mass: $292 \mathrm{~g}$, the center of gravity $L_{G}: 363 \mathrm{~mm}$ from the butt end) and a conventional weight and weight balanced racket (349 g, $L_{G}: 323 \mathrm{~mm}$ ) are selected as representatives. They are the super-large sized rackets made of carbon graphite with a head size of 120 square inches and the same geometry. The result showed that the shock vibration of the super-light weight balanced racket with super-large sized head is much larger than that of the conventional weight balanced type racket. It also showed that the sweet area of the former in terms of the shock vibration shifts from the center to the topside on the racket face compared to the latter. This is because the location of the grip on the racket handle is further from the location of the node on the handle of the first mode of super-light racket than that of the conventional weight racket.
\end{abstract}

Key words: Dynamics, Sports Engineering, Impact, Tennis Racket, Shock Vibrations, Wrist Joint, Super Large Sized Racket, Weight Distribution, Experimental Modal Analysis

\section{Introduction}

The implementation of material composites has led to increased flexibility in the design and production of sporting goods. The increased freedom has enabled manufacturers to tailor goods to match the different physical characteristics and techniques of users. However, ball and racket impact in tennis is an instantaneous non-linear phenomenon creating frame

${ }^{*}$ Received 17 Nov., 2009 (No. 09-0698) [DOI: 10.1299/jsdd.4.331]

Copyright $\odot 2010$ by JSME 
vibrations and large deformations in the ball/string system. The problem is further complicated by the involvement of humans in the actual strokes. Therefore, there are many unknown factors involved in the mechanisms explaining how the racket frame influences the racket capabilities.

The lightweight racket with handle-light configuration and large head size is recent tendency of high-tech tennis rackets, increasing power or post-impact ball velocity with an increasing racket swing speed.

This paper investigates the performance of lightweight tennis racket with super-large sized head in terms of feel or comfort. It predicts the effect of the mass and mass distribution of super-large sized rackets on the impact shock vibrations of the racket handle and the player's wrist joint when a player hits a flat forehand drive. The prediction is based on the identification of the racket characteristics, the damping of the racket-arm system, equivalent mass of the player's arm system and the approximate nonlinear impact analysis in tennis. The racket called EOS120A is employed as a representative example of a super-light racket (mass: $292 \mathrm{~g}$ including the weight of strings, the center of gravity $L_{G}: 363 \mathrm{~mm}$ from the butt end), while the racket called EOS120H is selected as a representative of a conventional weight and weight balanced racket $\left(349 \mathrm{~g}, L_{G}: 323 \mathrm{~mm}\right)$. They are the super-large sized rackets made of carbon graphite with a head size of 120 square inches and the same geometry.

\section{Method to Predict the Shock Transmitted to the Arm from Racket during Impact $^{(1)-(3)}$}

Figure 1 shows an impact model for the prediction of shock forces transmitted to the arm joints from a racket. The impact force $S_{0}$ at $\mathrm{P}_{0}$ causes a shock force $S_{I}$ on the player's hand $\mathrm{P}_{1}$, a shock force $S_{2}$ on the elbow $\mathrm{P}_{2}$, and finally a shock force $S_{3}$ on the player's shoulder $\mathrm{P}_{3}$ during the impact at which the player hits the ball with his racket.Since the intensity of the impulse decreases with the distance from the point of impact with the ball, it can be assumed that the shoulder does not basically alter its velocity, despite the presence of the shock force $S_{3}$. Generally speaking, the shock forces $S_{0}, S_{1}, S_{2}$, and $S_{3}$, which are mainly responsible for the sudden changes in velocity that take place in the brief interval of time considered, is one order of magnitude higher than the other forces in play during the same interval; consequently the gravity force and muscular action are not taken into account. Accordingly, we consider the racket to be freely hinged to the forearm of the player, the forearm being freely hinged to the arm and the arm freely hinged to the player's body.

This schematization only refers to the interval lasting no longer than one hundredth of a second: both before and afterwards, in the absence of shock forces $S_{0}, S_{1}, S_{2}$, and $S_{3}$, all the movements depend on the intensity of the muscular forces and gravity forces in play.

Let the forearm length be $a_{a}=\mathrm{P}_{1} \mathrm{P}_{2}$, with a mass $m^{\prime}$ to which the mass $m^{\prime \prime}$ of the hand is added: consequently, the total mass $m_{a}$ of the forearm is equal to $m_{a}=m^{\prime}+m^{\prime \prime}$ concentrated at $\mathrm{P}_{1}$, and the distance of the center of mass from the elbow be $b_{a}$. Moreover, let the moment
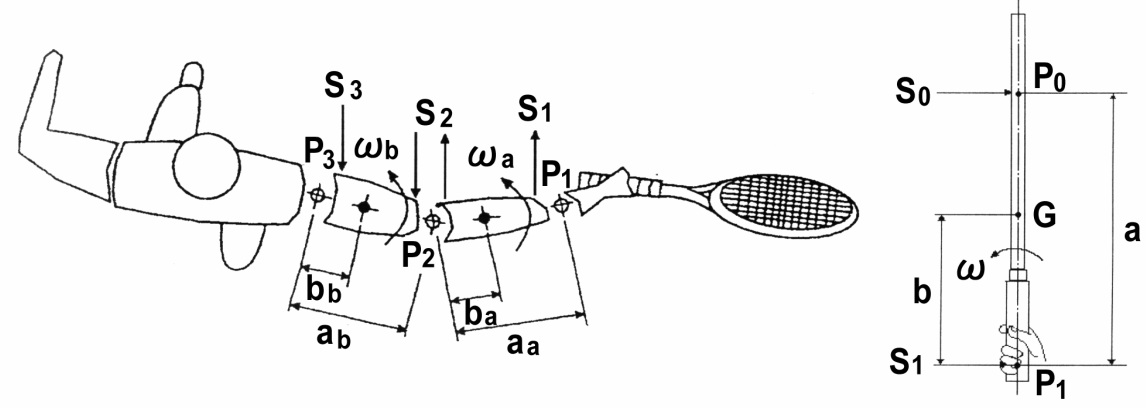

Fig. 1 Impact model for the prediction of the shock force transmitted to the arm joints from a racket. 
of inertia around the elbow $\mathrm{P}_{2}$ be $J_{a}$, the mass of the arm with a length of $a_{b}=\mathrm{P}_{2} \mathrm{P}_{3}$ be $m_{b}$, the distance of the center of mass from the shoulder $\mathrm{P}_{3}$ be $b_{b}=\mathrm{G}_{3} \mathrm{P}_{3}$, while the moment of inertia with respect to the shoulder $\mathrm{P}_{3}$ be $J_{b}$. We can derive the relationship between the shock acceleration $A_{n v}=\mathrm{d} V_{1} / \mathrm{d} t$ of point $\mathrm{P}_{1}$ and the shock force $S_{l}$ as follows. We will consider the very frequent case during the impact phase with the ball when the arm and forearm are aligned with one other, and $S_{1}$, orthogonal to axis $\mathrm{P}_{1} \mathrm{P}_{2} \mathrm{P}_{3}$, is the thrust the racket transmits to the hand. If $\omega_{a}$ and $\omega_{b}$ are the angular speed of the arm and forearm, the speed $V_{1}$ of point $\mathrm{P}_{1}$, where thrust $S_{I}$ acts, is equal to:

$$
V_{1}=\omega_{a} a_{a}+\omega_{b} a_{b}+V_{3}
$$

where $V_{3}$ is the speed of the center $\mathrm{P}_{3}$ of the shoulder articulation (joint), which is assumed to remain unchanged following the impulse mensioned above.

With the sign convention shown in Fig. 1 for the arm we have the two following equations of dynamic equilibrium:

$$
\begin{aligned}
& S_{2} a_{b}+J_{b} \mathrm{~d} \omega_{b} / \mathrm{d} t=0 \\
& S_{3}+S_{2}+m_{b} b_{b} \mathrm{~d} \omega_{b} / \mathrm{d} t=0
\end{aligned}
$$

from which it is easily determined that:

$$
\begin{array}{r}
\mathrm{d} \omega_{b} / \mathrm{d} t=-S_{2} a_{b} / J_{b} \\
S_{3} / S_{2}=m_{b} a_{b} b_{b} / J_{b}-1
\end{array}
$$

For the forearm we have the following equations of dynamic equilibrium:

$$
\begin{aligned}
& S_{l} a_{a}-J_{a} \mathrm{~d} \omega_{a} / \mathrm{d} t-m_{a} a_{b} b_{a} \mathrm{~d} \omega_{b} / \mathrm{d} t=0 \\
& S_{2}+S_{1}-m_{a}\left(a_{b} \mathrm{~d} \omega_{b} / \mathrm{d} t+b_{a} \mathrm{~d} \omega_{a} / \mathrm{d} t\right)=0
\end{aligned}
$$

and keeping account of Equation (3), after a few calculation steps we then obtain:

$$
\begin{gathered}
S_{2} / S_{I}=\chi_{a} \\
\mathrm{~d} \omega_{a} / \mathrm{d} t=\mu_{a} S_{I} a_{a} / J_{a}
\end{gathered}
$$

where

$$
\begin{aligned}
& \mu_{a}=\left[1+\left(m_{a} a_{b}{ }^{2} / J_{b}\right)\left(1-b_{a} / \mathrm{a}_{\mathrm{a}}\right)\right] /\left[1+\left(m_{a} a_{b}{ }^{2} / J_{b}\right)\left(1-m_{a} b_{a}{ }^{2} / J_{a}\right)\right] \\
& \chi_{a}=\left(m_{a} a_{a} b_{a} / J_{a}-1\right) /\left[1+\left(m_{a} a_{b}{ }^{2} / J_{b}\right)\left(1-m_{b} b_{a}{ }^{2} / J_{a}\right)\right]
\end{aligned}
$$

so that for Equations (1) and (7) we obtain:

$$
\begin{gathered}
A_{n v}=\mathrm{d} V_{1} / \mathrm{d} t=\mathrm{d}\left(\omega_{a} a_{a}+\omega_{b} a_{b}+V_{3}\right) / \mathrm{d} t=a_{a} \mathrm{~d} \omega_{a} / \mathrm{d} t+a_{b} \mathrm{~d} \omega_{b} / \mathrm{d} t \\
=\left[\mu_{a} a_{a}{ }^{2} / J_{a}-\chi_{a} a_{b}{ }^{2} / J_{b}\right] S_{l}
\end{gathered}
$$

i.e. by assuming 


$$
M_{H}=1 /\left[\mu_{a} a_{a}{ }^{2} / J_{a} \quad-\chi_{a} a_{b}^{2} / J_{b}\right]
$$

finally we have the shock acceleration $A_{n v}$ at the grip portion and the wrist joint as

$$
A_{n v}=\mathrm{d} V_{1} / \mathrm{d} t=S_{1} / M_{H}
$$

From Eq.(9) we can deduce that the inertia effect of the arm and the forearm can be attributed to a mass $M_{H}$ concentrated in the hand: therefore the analysis of impact between ball and racket can be carried out by assuming that the racket is free in space, as long as the mass $M_{H}$ is applied at point $\mathrm{P}_{1}$ on the racket handle.

If the impact force $S_{0}$ between a ball and the racket is given when the ball hits the racket, the shock force $S_{l}$ and the angular acceleration $\mathrm{d} \omega / \mathrm{d} t$ can be obtained from the equations of dynamic equilibrium:

$$
\begin{aligned}
& S_{0} a+J \mathrm{~d} \omega / \mathrm{d} t+M_{R} b \mathrm{~d} V_{1} / \mathrm{d} t=0 \\
& S_{0}+M_{R} b \mathrm{~d} \omega / \mathrm{d} t+\left(M_{R}+M_{H}\right) \mathrm{d} V_{1} / \mathrm{d} t=0
\end{aligned}
$$

with a few steps we obtain:

$$
\begin{gathered}
\mathrm{d} \omega / \mathrm{d} t=-\mu a S_{0} / J \\
S_{1}=M_{H} \mathrm{~d} V_{1} / \mathrm{d} t=\chi S_{0}
\end{gathered}
$$

where:

$$
\begin{gathered}
\mu=\left[1+\left(M_{R} / M_{H}\right)(1-b / a)\right] /\left(1+\left(M_{R} / M_{H}\right)\left(1-M_{R} b^{2} / J\right)\right. \\
\chi=\left(M_{R} a b / J-1\right) /\left[1+\left(M_{R} / M_{H}\right)\left(1-M_{R} b^{2} / J\right)\right]
\end{gathered}
$$

where we let the mass of the racket be $M_{R}$, the distance between the grip location on the handle and the impact location on the string face be $a$, the distance between the grip location on the handle and the center of mass of the racket be $b$, and the moment of inertia with respect to the articulation $\mathrm{P}_{1}$ of the hand be $J^{(1)-(3)}$.

\section{Method to Predict the Restitution Coefficient and Impact Force between} Ball and Racket-Arm System

\subsection{Reduced Mass of a Racket and a Racket-Arm System}

We introduce the reduced mass $M_{r}$ of a racket at the impact location on the racket face in order to make the impact analysis simpler. It can be derived from the principle of the conservation of angular momentum if the moment of inertia and the distance between an impact location and a center of gravity are given. Consider a ball that impacts the front of a racket at a velocity of $V_{B O}$ and also assume that the racket after impact rotates around the center of gravity, which moves along a straight line. The impulse $\int_{F}(t) d t$ could be described as the following equations, in case the pre-impact velocity $V_{G 0}=0$ for simplicity, where $F(t)$ is the impact force, $m_{B}$ is the mass of a ball, $V_{B}$ is the post-impact velocity of a ball, $M_{R}$ is the mass of a racket, $V_{G}$ is the post-impact velocity of the center of gravity.

$$
\int F(t) d t=m_{B} V_{B o}-m_{B} V_{B}
$$




$$
\int F(t) d t=M_{R} \cdot V_{G}
$$

The following equation can be expressed if the law of angular momentum conservation is applied, in case pre-impact angular velocity $\omega_{0}=0$, where the distance $c_{0}$ between the center of gravity and the impact location, the inertial moment $I_{G O X}$ around the center of gravity, and the angular velocity $\omega$ immediately after impact are given.

$$
\int F(t) d t \cdot c_{o}=I_{G O X} \cdot \omega
$$

Based on the geometric relationships, the velocity $V_{R}$ at the impact location of the racket after impact can be expressed as follows:

$$
V_{R}=V_{G}+\omega c_{0}
$$

When $\omega$ and $V_{G}$ are eliminated, the following equation can be written:

$$
\left(\frac{I_{G 0 X} M_{R}}{I_{G 0 X}+M_{R} c o^{2}}\right) V_{R}=m_{B}\left(V_{B 0}-V_{B}\right)
$$

Thus, we can express the law of conservation of linear momentum as

$$
m_{B} V_{B 0}=m_{B} V_{B}+M_{r} V_{R}
$$

where,

$$
M_{r}=\frac{I_{G 0 X} M_{R}}{I_{G 0 X}+M_{R} c_{0}^{2}}
$$

The symbol $M_{r}$ refers to the reduced mass at the impact location for a racket freely suspended. Thus the motion of a racket as a rigid body could be analyzed as though the racket were a particle. The inertial moment $I_{G O X}$ is obtained using $T_{X}$; the measured pendulum vibration period, $g_{2}$ the gravity acceleration, $d_{i}$ the distance between the support location and the center of gravity of a racket as a physical pendulum.

$$
I_{G 0 X}=\left(\frac{T_{X}}{2 \pi}\right)^{2} M_{R} g d-M_{R} d^{2}
$$

The shock forces during impact are assumed to be one order of magnitude higher than those due to gravity and muscular action. Accordingly, we consider the racket to be freely hinged to the forearm of the player, the forearm being freely hinged to the arm and the arm freely hinged to the player's body. We can deduce that the inertia effect of the arm and the forearm can be attributed to a mass $M_{H}$ concentrated in the hand; therefore the analysis of impact between ball and racket can be carried out by assuming that the racket is free in space, as long as the mass $M_{H}$ is applied at the hand grip as previously described. The reduced mass $M_{r}$ at the impact location with a racket-arm system can be derived as

$$
M_{r}=\frac{I_{G M X}\left(M_{R}+M_{H}\right)}{I_{G M X}+\left(M_{R}+M_{H}\right) c^{2}}
$$

where 


$$
\begin{gathered}
c=c_{o}+\left(L_{G o}-L_{H}\right) M_{H} /\left(M_{R}+M_{H}\right) \\
I_{G M X}=I_{G o X}+M_{R} \triangle G^{2}+M_{H}\left(L_{G o}-L_{H}-\triangle G\right)^{2} \\
\triangle G=\left(L_{G o}-L_{H}\right) M_{H} /\left(M_{R}+M_{H}\right)
\end{gathered}
$$

and $L_{G o}$ denotes the distance between the center of mass and the grip end of the racket, $I_{G o X}$ the moment of inertia with respect to the center of gravity of the racket, $c_{o}$ the distance between the center of gravity of the racket and the impact location, and $L_{H}$ the distance of the location of the player's hand from the grip end. The moment of inertia with respect to the center of gravity and the distance of the center of gravity of the racket-arm system from the impact location are indicated by $I_{G M X}$ and $c$, respectively.

\subsection{Restoring Force Characteristics and Enegy Loss of a Ball-String System}

Figure 2 shows schematically the test for obtaining the applied force- deformation curves, where the ball is deformed between two flat surfaces as shown in (a) and the ball plus strings is deformed with a racket head clamped as shown in (b). The results for the ball and racket are shown in Fig.3. According to the pictures of a racket being struck by a ball, it seems that the ball deforms only at the side, which contact to the strings ${ }^{(5)}$.

Assuming that a ball with concentrated mass deforms only at the side in contact with the strings ${ }^{(7)}$, the curves of restoring force $F_{\mathrm{B}}$ vs. ball deformation, restoring force $F_{\mathrm{G}}$ vs. strings deformation, and the restoring force $F_{\mathrm{GB}} \mathrm{Vs}$. deformation of the composed ball/strings system are obtained from Fig. 3 as shown in Fig.4. These restoring characteristics are determined in order to satisfy a number of experimental data using the least square method. The curves of the corresponding stiffness $K_{\mathrm{B}}, K_{\mathrm{G}}$ and $K_{\mathrm{GB}}$ are derived as shown in Fig.5 by differentiation of the equations of restoring force with respect to deformation. The stiffness $K_{\mathrm{B}}$ of a ball, $K_{\mathrm{G}}$ of strings and $K_{\mathrm{GB}}$ of a composed ball/strings system exhibit strong nonlinearity.

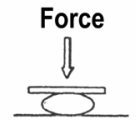

(a) a ball

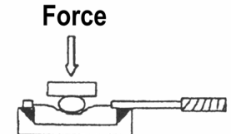

(b) a composed ball / string system

Fig.2 Illustrated applied forcedeformation test

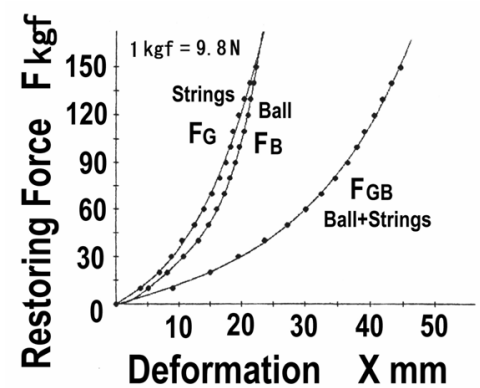

Fig.4 Restoring forces vs. deformation of a ball, strings, and a Composed ball/string system assuming that a ball deforms only at the side in contact with the strings.

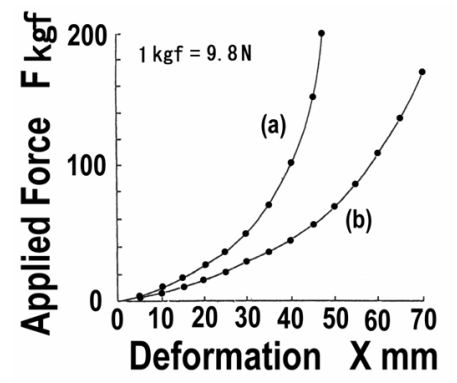

Fig.3 Results of a force-deformation test. 
The measured coefficient of restitution versus the incident velocity when a ball strikes the rigid wall is shown in Fig.6, while the measured coefficient of restitution $e_{\mathrm{BG}}$, which is abbreviated as COR, when a ball strikes the strings with a racket head clamped is shown in Fig.7. Although the COR in Fig. 6 decreases with increasing incident velocity, the coefficient $e_{\mathrm{BG}}$ with a racket head clamped is almost independent of ball velocity and strings tension. This value of COR can be regarded as being inherent to the materials of ball and strings, showing the important role of strings. This feature is due to the nonlinear restoring force characteristics of a composed ball/strings system $^{(4)}$.

Since equivalent spring stiffness $K_{G B}$ of the compound system increases as the impact velocity increases, the independence of the damping coefficient ratio with respect to impact velocity means that damping coefficient $C_{G B}$ is proportional to $K_{G B}{ }^{1 / 2}$ and increases with increases in impact velocity in Fig.8. The energy loss of a ball and strings due to impact can be related to the coefficient $e_{B G}$

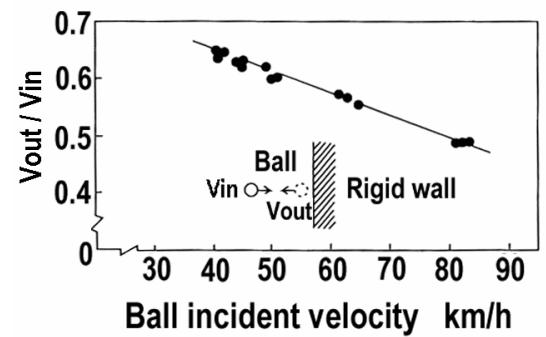

Fig.6 Measured coefficient of restitution (COR) between a ball and a rigid wall.

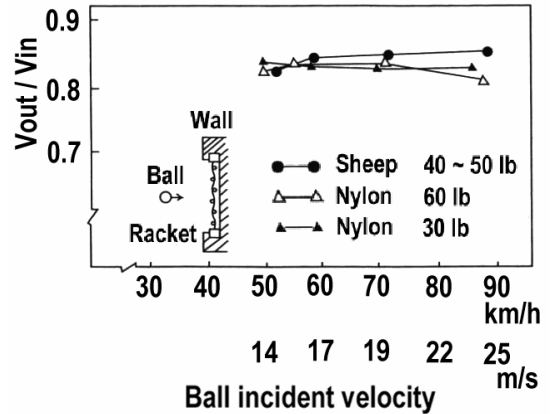

(a)

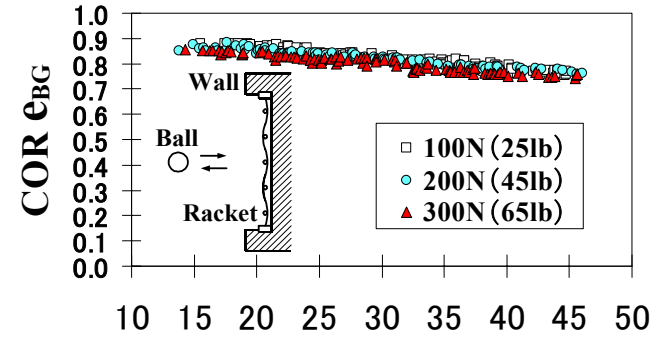

Impact velocity $[\mathrm{m} / \mathrm{s}]$

(b)

Fig.7. Measured $\operatorname{COR} e_{B G}$ between a ball and strings with frame clamped.

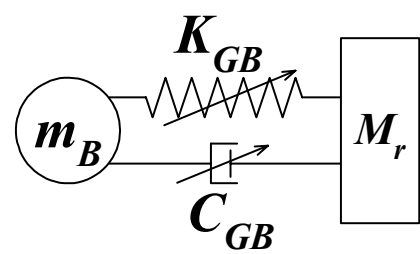

Fig.8 Non-linear impact model of a ball- string system.

The result of measured contact time, which means how long the ball stays on the strings, with a normal racket and with a wide-body racket (stiffer) shows that the stiffness of the racket frame does not affect the contact time much ${ }^{(4)}$. Accordingly, the masses of a ball and a racket as well as the nonlinear stiffness of a ball and strings are the main factors in the deciding of a contact time. Therefore, the contact time can be calculated using a model assuming that a ball with a concentrated mass $m_{B}$ and a nonlinear spring $K_{B}$, collides with the nonlinear spring $K_{G}$ of strings supported by a frame without vibration, where the measured coefficient of restitution inherent to the materials of ball-strings impact is employed as one of the sources of energy loss. 


\subsection{Method to Predict the Restitution Coefficient Between a Ball and a Racket}

In case the vibration of the racket frame is neglected, the momentum equation and the measured coefficient restitution $e_{G B}$ give the approximate post-impact velocity $V_{B}$ of a ball and $V_{R}$ of a racket at the impact location. The impulse could be described as the following equation using the law of conservation of linear momentum and the equation for the velocity difference with the coefficient of restitution, where $m_{B}$ is the mass of a ball, $M_{r}$ is the reduced mass of a racket-arm system at the hitting location, and $V_{B o}$ and $V_{R o}$ are the ball velocity and racket head velocity before impact, respectively.

$$
\int F(t) d t=m_{B} V_{B o}-m_{B} V_{B}=\left(V_{B O}-V_{R o}\right)\left(1+e_{G B}\right) m_{B} /\left(1+m_{B} / M_{r}\right)
$$

Assuming the contact duration during impact to be half the natural period of a whole system composed of $m_{B}, K_{G B}$ and $M_{r}$ as shown in Fig.8, the approximate contact time $T_{c}$ could be obtained according to the vibration theory.

$$
T_{c}=\pi m_{B}^{1 / 2} /\left(K_{G B}\left(1+m_{B} / M_{r}\right)^{1 / 2}\right.
$$

In order to make the analysis simpler, the approximate equivalent force $F_{\text {mean }}$ can be introduced during contact time $T_{c}$, which is described as

$$
\int{ }^{T_{c}} F(t) d t=F_{\text {mean }} \cdot T_{c}
$$

Thus, from Eq.(27), Eq.(28) and Eq.(29), the relationship between $F_{\text {mean }}$ and corresponding $K_{G B}$ against the pre-impact velocity $\left(V_{B O}-V_{R o}\right)$ is given by

$$
F_{\text {mean }}=\left(V_{B O}-V_{R o}\right)\left(1+e_{G B}\right) m_{B}{ }^{1 / 2} K_{G B}{ }^{1 / 2} / \pi\left(1+m_{B} / M_{r}\right)^{1 / 2}
$$

where $e_{B G}$ is the measured coefficient of restitution when a ball strikes the clamped string bed for estimating energy loss of the ball and the strings, being equivalent to the nonlinear damping coefficient $C_{\mathrm{GB}}$ in Fig.8.

On the other hand, the non-linear relationship between the measured restoring force $F_{\mathrm{GB}}$ (Fig.4) vs. stiffness $K_{\mathrm{GB}}$ (Fig.5) of the composed strings/ball system can be expressed in the form

$$
F_{\text {mean }}=f\left(K_{G B}\right) .
$$

From Eq.(30) and Eq.(31), $K_{G B}$ and $F_{\text {mean }}$ against the pre-impact velocity can be obtained, accordingly $T_{C}$ can also be determined against the pre-impact velocity ${ }^{(5)}$. Figure 9 is a comparison between the measured contact times during actual forehand strokes ${ }^{(5)}$ and the calculated ones when a ball hits the center of the strings face of a conventional type racket, showing a good agreement. Since the force-time curve of impact has an influence on the magnitude of racket frame vibrations, it is approximated as a half-sine pulse, which is almost similar in shape to the actual impact force. The mathematical expression of approximate impact force is

$$
F(t)=F_{\max } \sin \left(\pi t / T_{c}\right) \quad\left(0 \leqq t \leqq T_{c}\right)
$$

where $F_{\text {max }}=\pi F_{\text {mean }} / 2$. The Fourier spectrum $S_{F}(f)$ of Eq.(32) is represented as

$$
S_{F}(f)=2 F_{\max } T_{c}\left|\cos \left(\pi f T_{c}\right)\right| /\left[\pi\left|1-\left(2 f T_{c}\right)^{2}\right|\right]
$$




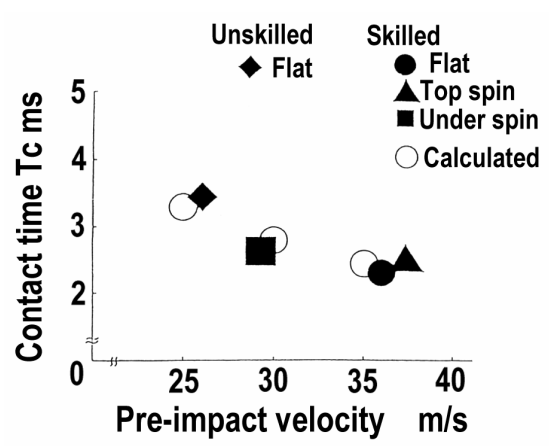

Fig.9 Comparison between the measured contact times during strokes and the calculated results.

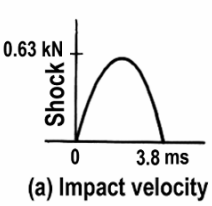

(a) Impact velocity

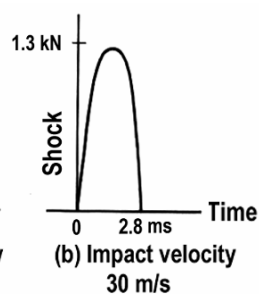

Fig.10 Calculated shock shape when a ball strikes the center on the String face at velocities of $20 \mathrm{~m} / \mathrm{s}$ and $30 \mathrm{~m} / \mathrm{s}$.

where $f$ is the frequency. Figure 10 shows the examples of the calculated shock shape during impact, where the ball strikes the center on the string face at a velocity of (a) $20 \mathrm{~m} / \mathrm{s}$ and (b) 30 $\mathrm{m} / \mathrm{s}$ with the racket strung at $55 \mathrm{lb}$, respectively.

The vibration characteristics of a racket can be identified using the experimental modal analysis ${ }^{(9)-(12)}$ and the racket vibrations can be simulated by applying the impact force-time curve to the hitting portion on the racket face of the identified vibration model of a racket. When the impact force $S_{F j}\left(2 \pi f_{k}\right)$ applies to the point $j$ on the racket face, the amplitude $X_{i j k}$ of $k$-th vibration mode component at point $i$ is expressed as

$$
X_{i j k}=r_{i j k} S_{F j}\left(2 \pi f_{k}\right)
$$

where $r_{i j k}$ denotes the residue of $k$-th mode between arbitrary point $i$ and $j$, and $S_{F j}\left(2 \pi f_{k}\right)$ is the impact force component of $k$-th frequency $f_{k}$. It is assumed that the ball contacts to the string face at the four cross points ${ }^{(12)}$.

The energy loss due to the racket vibration induced by impact can be derived from the amplitude distribution of the vibration velocity and the mass distribution along the racket frame. The coefficient of restitution $e_{r}(\mathrm{COR})$ can be derived considering the energy loss $E_{1}$ due to racket vibrations and $E_{2}$ due to large deformations of a ball and strings corresponding to the coefficient $e_{B G}$. If a ball collides with a racket at rest $\left(V_{R o}=0\right)$, the coefficient of restitution $e_{r}$ corresponding to the total energy loss $E\left(=E_{1}+E_{2}\right)$ can be obtained as

$$
e_{r}=\left(V_{R}-V_{B}\right) / V_{B O}=\left[1-2 E\left(m_{B}+M_{r}\right) /\left(m_{B} M_{r} V_{B O}^{2}\right)\right]^{1 / 2}
$$

The coefficient of damping $C_{G B}$ can be derived from the coefficient of restitution $e_{r}$ finally. Thus, the force-time curve of impact between a ball and a racket considering the vibrations of a racket frame can be approximated as

$$
S_{0}(t)=F(t)=F_{\text {max }} \sin \left(\pi t / T_{c}\right) \quad\left(0 \leqq t \leqq T_{c}\right)
$$

where

$$
F_{\text {max }}=\left(\pi /\left(2 T_{c}\right)\right)\left(V_{B O}-V_{R o}\right)\left(1+e_{r}\right) m_{B} /\left(1+m_{B} / M_{r}\right)
$$

The impact force $F(t)$ is equivalent to the impact force $S_{0}$ in Fig.1.

The post-impact ball velocity $V_{B}$ is represented as

$$
V_{B}=-V_{B o}\left(e_{r}-m_{B} / M_{r}\right) /\left(1+m_{B} / M_{r}\right)+V_{R o}\left(1+e_{r}\right) /\left(1+m_{B} / M_{r}\right)
$$

Accordingly, if the ratio of rebound velocity against the incident velocity of a ball when a ball strikes the freely suspended racket ( $V_{R o}=0$ ) is defined as the rebound power coefficient $e$, it is 
written as Eq.(39). The rebound power coefficient is often used to estimate the rebound power performance of a racket experimentally in the laboratory.

$$
e=-V_{B} / V_{B O}=\left(e_{r}-m_{B} / M_{r}\right) /\left(1+m_{B} / M_{r}\right)
$$

When a player hits a coming ball with a pre-impact racket head velocity $V_{R o}$, the coefficient $e$ can be expressed as

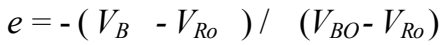

Figure 11 is a comparison between the measured $e$ and the predicted $e$ when a ball hit a freely-suspended racket (about $30 \mathrm{~m} / \mathrm{s}$ ), showing a good agreement between them ${ }^{(4)-(6)}$.

The power of the racket could be estimated by the post-impact ball velocity $V_{B}$ when a player hits the ball. The $V_{B}$ can be expressed as Eq.(41). The $V_{R o}$ is given by $L_{X}\left(\pi N_{s} / I_{S}\right)^{1 / 2}$, where $L_{X}$ denotes the horizontal distance between the player's shoulder joint and the impact location on the racket face, $N_{s}$ the constant torque about the shoulder joint, and $I_{s}$ the moment of inertia of arm/racket system about the shoulder joint. Figure 12 shows a simple forehand ground stroke swing model ${ }^{(9),(10)}$.

$$
V_{B}=-V_{B o} e+V_{R o}(1+e)
$$

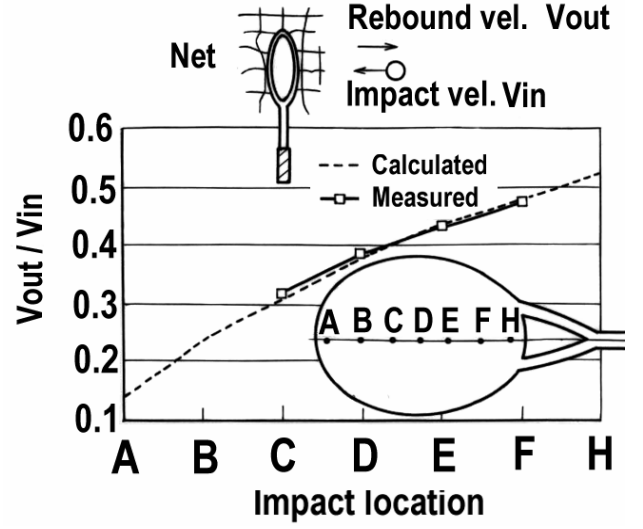

Fig.11 Comparison between the measured rebound power coefficient $e$ and the predicted one $\left(V_{B}\right.$ $=V_{\text {out }}, V_{B O}=V_{\text {in }}, V_{R o}=0$ ).

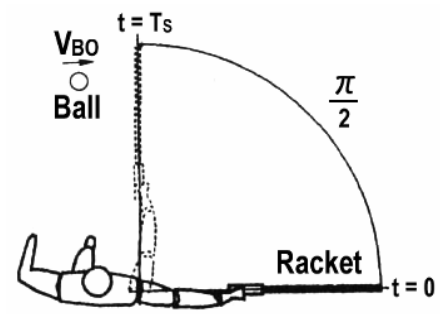

Fig.12 Forehand stroke model

\section{Method to Predict the Shock Accelerations Transmitted to the Arm from} Racket during Impact

The shock acceleration $A_{n v}(t)$ at the hand grip considering the equivalent mass $M_{H}$ of the arm system when a ball collied with a suspended racket for simplicity $\left(V_{R o}=0\right)$ can be represented, from

$S_{0}(t)=\left(M_{R}+M_{H}\right)\left(\mathrm{d} V_{\mathrm{G}} / \mathrm{d} t\right), \quad S_{0}(t) a=I_{G}(\mathrm{~d} \omega / \mathrm{d} t), \quad \mathrm{d} V_{1} / \mathrm{d} t=\left(\mathrm{d} V_{\mathrm{G}} / \mathrm{d} t\right)-X(\mathrm{~d} \omega / \mathrm{d} t)$,

as

$$
A_{n v}(t)=\mathrm{d} V_{1} / \mathrm{d} t=S_{0}(t)\left[1 /\left(M_{R}+M_{H}\right)-\left(a / I_{G}\right) X\right]
$$

where $X$ denotes the distance between the center of mass of the racket-arm system and the location of the player's hand, $a$ the distance between the center of mass of racket-arm system and the impact location of the racket, $I_{G}$ the moment of inertia around the center of mass of racket-arm system, respectively. The maximum shock force $S_{1 \max }$ transmitted to a wrist joint corresponds to the maximum impact force $S_{0 \max }$. 


\section{Method to Predict the Vibration Component at the Racket handle and the Wrist Joint}

The natural frequency of racket frame drops slightly and the position of the node on the handle shifts somewhat to the held position for the hand-held racket compared to the freely suspended racket. Furthermore the damping of frame vibrations is remarkably larger for the hand-held racket compared to the freely suspended racket. Nevertheless, there is no big difference in the initial amplitude distributions of a racket frame between the hand-held racket and the freely suspended racket. The vibration displacement component $X_{i j, k}(t)$ of $k$-th mode at the location $i$ of the racket handle or the wrist joint is represented as

$$
X_{i j, k}(t)=r_{i j k} S_{0 j}\left(2 \pi f_{k}\right) \exp \left(-2 \pi f_{k} \zeta_{k} t\right) \sin \left(2 \pi f_{k} t\right)
$$

Accordingly, the vibration acceleration component $A_{i j, k}(t)$ of $k$-th mode at the location $i$ of the racket handle or the wrist joint is represented as

$$
A_{i j, k}(t)=-\left(2 \pi f_{k}\right)^{2} r_{i j k} S_{0 j}\left(2 \pi f_{k}\right) \exp \left(-2 \pi f_{k} \zeta_{k} t\right) \sin \left(2 \pi f_{k} t\right)
$$

where $j$ denotes the impact location between ball and racket on the string face, $\zeta_{k}$ the damping ratio of $k$-th mode with hand-held racket $\zeta_{\mathrm{H} 1}$ or wrist joint $\zeta_{\mathrm{w} 1}, S_{0 j}\left(2 \pi f_{k}\right)$ the fourier spectrum of Eq.(36). The summation of Eq.(42) and Eq.(43) represents the shock vibrations at the racket handle or the wrist joint.

Figure 13 shows an experiment where a male tournament player hits a flat forehand drive and Fig.14 shows the locations of attached accelerometers at the wrist joint and the elbow joint in the experiment. Figure 15 shows the center of gravity in a racket-arm system. Figure 16 is the result of the predicted accelerations of the shock vibrations of a wrist joint compared with the experimental ones when a ball is struck at the topside of the racket face. The predicted wave form was obtained using Eq.(42) and Eq.(43) as follows. The racket is

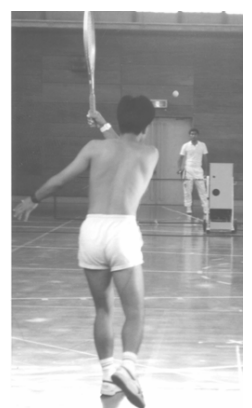

Fig. 13 Experiment where a male player hits flat forehand drive

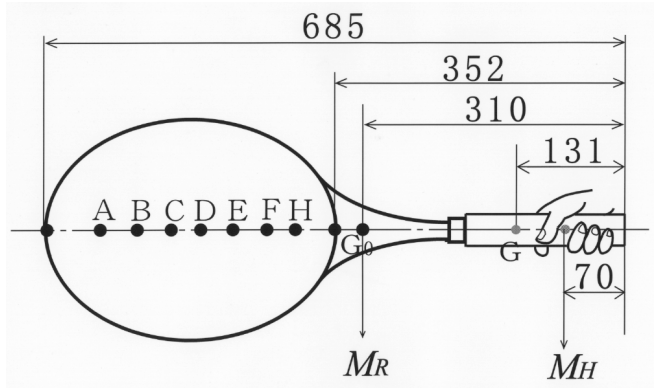

Fig. 15 Center of gravity in a racket-arm system (Racket:IMP-3 )

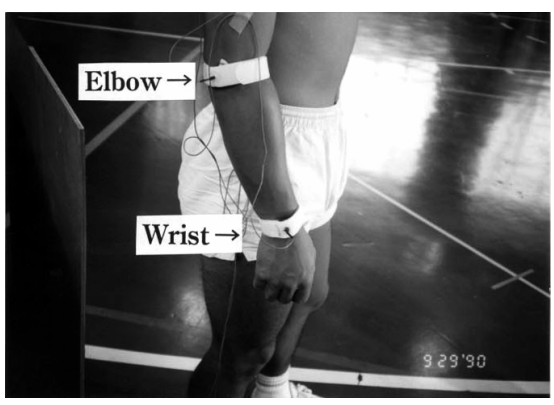

Fig. 14 Accelerometers attached at the wrist and the elbow.

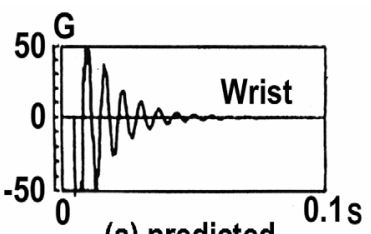

(a) predicted

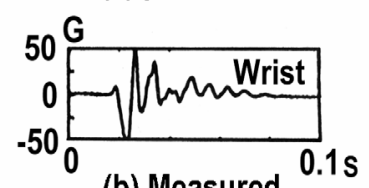

(b) Measured

Fig.16 Predicted shock vibrations of a wrist joint compared with the experimental. 
made of $75 \%$ graphite, $20 \%$ fiberglass and $5 \%$ others, with $685 \mathrm{~mm}$ of total length, $100 \mathrm{in}^{2}$ of face area, $342 \mathrm{~g}$ of mass including string mass, $310 \mathrm{~mm}$ of the center of mass from grip end, $14.2 \mathrm{gm}^{2}$ of moment of inertia about the center of racket mass, $60 \mathrm{lbs}$. of strings tension. The center of mass of racket-arm system shifts to the location of $131 \mathrm{~mm}$ from the grip end. The first largest peak in Fig.16 was caused by the initial shock and vibrations during the impact, followed by the residual vibrations of a racket frame. The shock vibrations are composed of the impact shock component and the vibration components, and each component has its own time history and magnitude depending on the impact velocity, impact location, grip location of racket handle and the physical properties of a racket. The damping ratio $\zeta_{\mathrm{H} 1}$ of a hand-held racket during actual impact has been estimated as about 2.5 times that of the one identified by the experimental modal analysis with small vibration amplitude (1st mode: $\left.\zeta_{\mathrm{Fl}}=0.024\right)$. Furthermore, the damping $\zeta_{\mathrm{w} 1}$ of the waveform at the wrist joint has been 3.0 times that at the grip portion of the racket handle. The predicted waveform of the shock vibrations with the wrist joint agrees fairly well with the measured one during actual forehand stroke by a player ${ }^{(2)(4)(11)}$. Average data taken from several scientific literatures with the weight and the height of a player were $60 \mathrm{kgw}$ and $170 \mathrm{~cm}$, respectively. The mass $M_{H}$ concentrated in the hand was estimated as $M_{H}=0.97 \mathrm{~kg}$, nearly 1.0 $\mathrm{kg}$.

The rebound power coefficient $e\left(=-V_{B} / V_{B O}\right)$ of a handled racket was agreed with that of the same racket with player's reduced mass on the handle in the laboratory tests ${ }^{(13)}$.

6. Predicted Impact Shock Vibrations at Tennis Player's Wrist Joint: Comparison between Two Super Large Sized Rackets with Big Difference in Frame Weight Distribution

\subsection{Comparison of Racket Physical Properties}

Figure 17 shows the super-large sized rackets made of carbon graphite with a head size of 120 square inches. Table 1 shows the measured racket physical properties, where the sign $I_{G Y}$ denotes the moment of inertia about the center of mass, the $I_{G R}$ the moment of inertia about the grip portion $70 \mathrm{~mm}$ from the grip end, the $I_{G X}$ the moment of inertia about the longitudinal axis of racket head. The reduced mass is at the center of racket face.

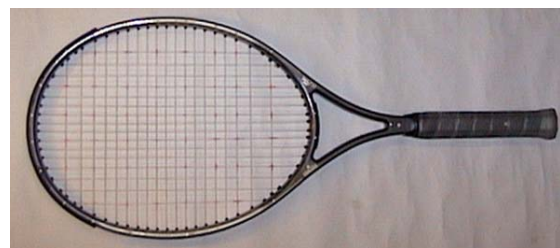

Fig.17 Super-large sized racket with a head size of 120 square inches.
Table 1 Physical properties of rackets

\begin{tabular}{|c|c|c|}
\hline Racket & $\mathrm{EOS} 120 \mathrm{H}$ & EOS120A \\
\hline $\begin{array}{l}\text { Face } \\
\text { area }\end{array}$ & $\begin{array}{l}120 \\
\text { in }^{2} \\
\end{array}$ & $\begin{array}{l}120 \\
\text { in }^{2} \\
\end{array}$ \\
\hline $\begin{array}{r}\text { Total } \\
\text { length } \\
\end{array}$ & $\begin{array}{c}27 \mathrm{in} \\
(685 \mathrm{~mm}) \\
\end{array}$ & $\begin{array}{c}27 \mathrm{in} \\
(690 \mathrm{~mm}) \\
\end{array}$ \\
\hline $\begin{array}{c}\text { Mass } \\
\text { (+Strings) }\end{array}$ & $349 \mathrm{~g}$ & $292 \mathrm{~g}$ \\
\hline $\begin{array}{c}\text { Center of } \\
\text { Gravity }\end{array}$ & $323 \mathrm{~mm}$ & $363 \mathrm{~mm}$ \\
\hline $\mathrm{I}_{\mathrm{GY}}$ & $16.0 \mathrm{~g} \cdot \mathrm{m}^{2}$ & $14.0 \mathrm{~g} \cdot \mathrm{m}^{2}$ \\
\hline $\mathrm{I}_{\mathrm{GR}}$ & $38.0 \mathrm{~g} \cdot \mathrm{m}^{2}$ & $39.0 \mathrm{~g} \cdot \mathrm{m}^{2}$ \\
\hline $\mathrm{I}_{\mathrm{GX}}$ & $2.21 \mathrm{~g} \cdot \mathrm{m}^{2}$ & $1.78 \mathrm{~g} \cdot \mathrm{m}^{2}$ \\
\hline $\begin{array}{l}1 \text { st } \\
\text { freq }\end{array}$ & $142 \mathrm{~Hz}$ & $137 \mathrm{~Hz}$ \\
\hline $\begin{array}{l}\text { Strings } \\
\text { tension }\end{array}$ & $79 \mathrm{lbs}$ & $79 \mathrm{lbs}$ \\
\hline $\begin{array}{c}\text { Reduced } \\
\text { mass }\end{array}$ & $205 \mathrm{~g}$ & $206 \mathrm{~g}$ \\
\hline
\end{tabular}


Figure 18 shows the predicted maximum shock acceleration at the grip portion of (a) a freely suspended racket, (b) a hand-held racket (nearly $M_{H}=1.0 \mathrm{~kg}$ ) when a ball strikes the various locations on the string face along the longitudinal axis. The difference between two rackets are not so large, however, the reduced mass on the handle of a player decreases remarkably the shock acceleration at the wrist joint.

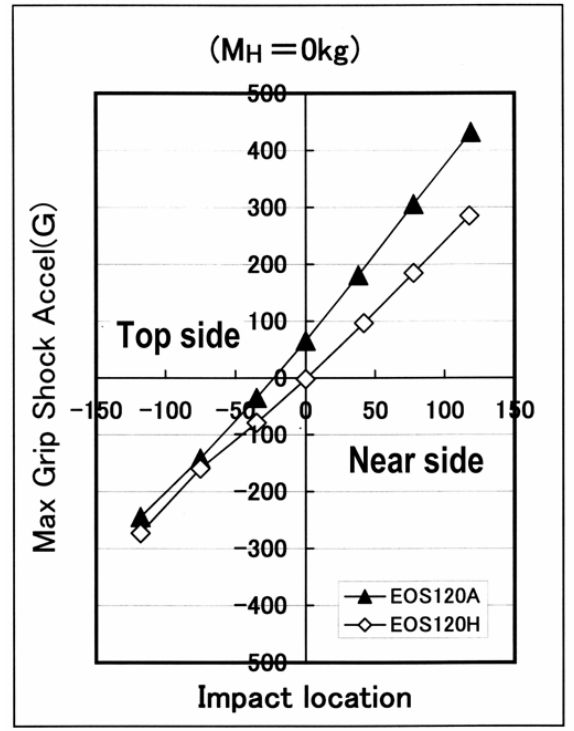

(a) a freely suspended racket

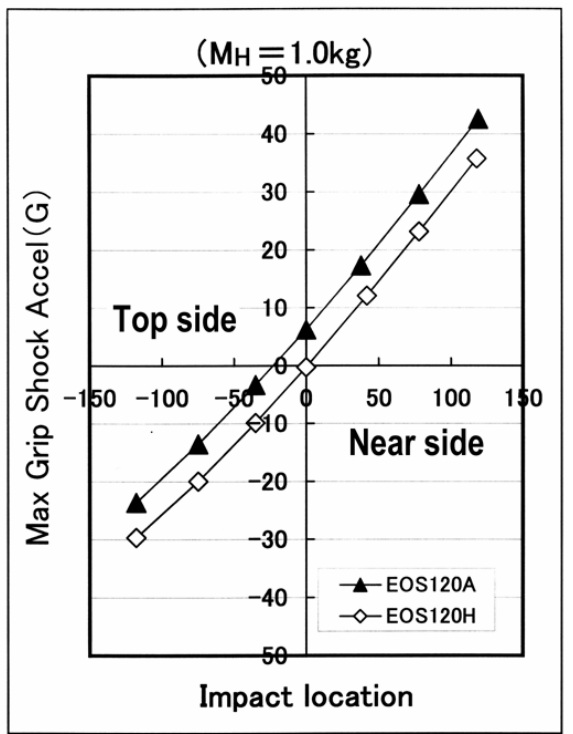

(b) a hand-held racket.

Fig.18 Predicted maximum shock acceleration at the grip (impact velocity: $30 \mathrm{~m} / \mathrm{s}$ ).

\subsection{Comparison of Initial Vibration Amplitude Components at the racket Handle}

Figure 19 shows the predicted vibration amplitude components during impact at the grip $70 \mathrm{~mm}$ from the grip end when a ball strikes the various locations on the string face along the longitudinal axis and along the short axes at the topside B, center D and nearside F, where the four vibration modes of freely suspended rackets are considered. The vibration of the super-light racket at the grip is much larger than that of the conventional weight balanced type racket. It is because the location $0.10 \mathrm{~L}$ (70 $\mathrm{mm}$ from the grip end) of grip on the handle is more apart from the location of node on the handle ( border line of black and white in Fig.20) of the first mode of super-light racket than that of the conventional weight balanced type racket.

It also shows that the sweet area with respect to the vibration is located around $30 \mathrm{~mm}$ topside from the center on the racket face with a super-light racket.

\subsection{Predicted Waveforms of Shock Vibrations at the Racket Handle}

Figure 21 shows the predicted waveform of the shock vibrations at the grip on comparing the two freely suspended rackets with different weight and weight balance when a ball strikes the various locations on the string face. The impact velocity between the ball and the racket is $30 \mathrm{~m} / \mathrm{s}$. The shock vibration of the super-light racket at the grip is much larger than that of the conventional weight balanced type racket.

\subsection{Predicted Waveforms of Shock Vibrations at the Wrist Joint}

The predicted waveform of the shock vibrations at the wrist joint agrees fairly well with the measured ones during actual forehand stroke by a player as seen in Fig. $16^{(2)(11)}$.

Figure 22 shows the predicted waveform of the shock vibrations of the player's wrist 

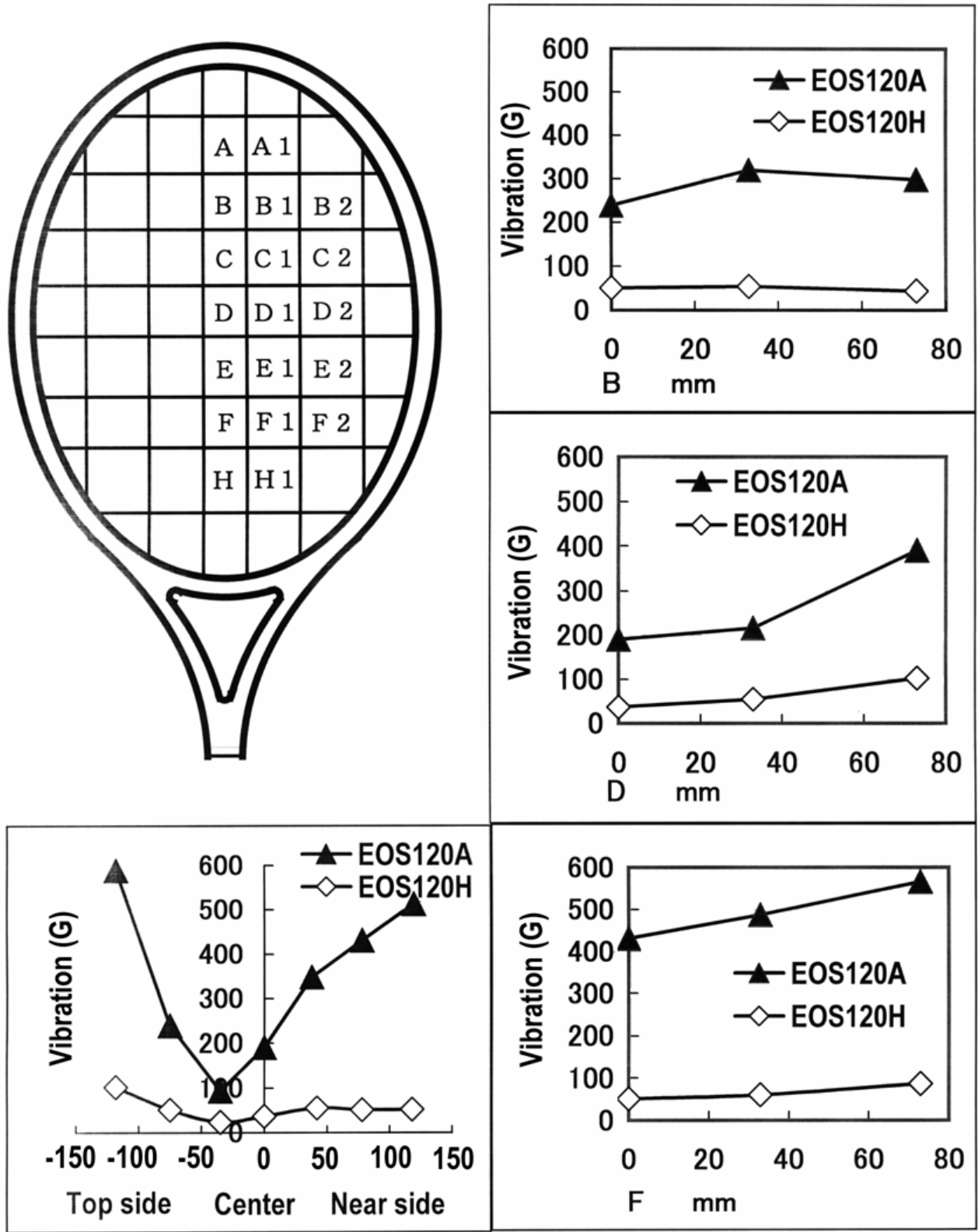

Fig. 19 Predicted maximum peak-peak vibration amplitudes components at the grip (impact velocity: $30 \mathrm{~m} / \mathrm{s}$ ) when a ball strikes the various locations on the string face of freely suspended rackets along the longitudinal axis and along the short axes at the topside $\mathrm{B}$, center $\mathrm{D}$ and nearside $\mathrm{F}$, where the four vibration modes of freely suspended rackets are considered.

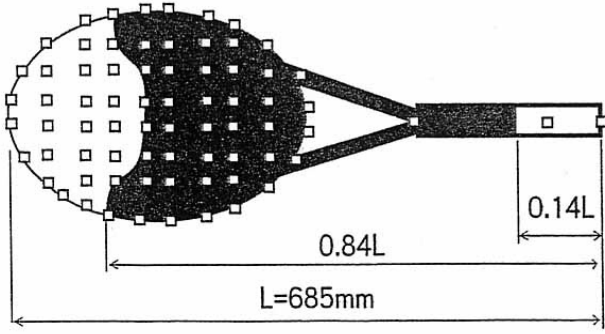

(a) EOS120H:Conventional weight distribution (349 g)

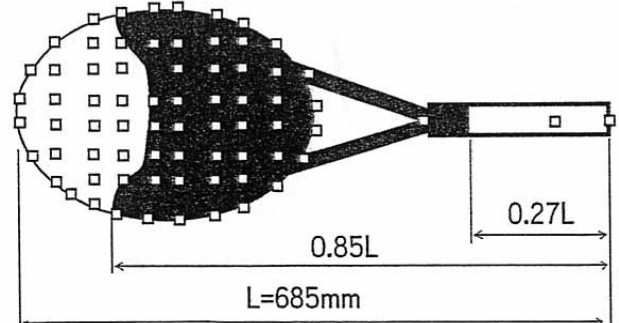

(b) EOS120A: Super-light top heavy $(292 \mathrm{~g})$

Fig.20 Locations of vibration node on the racket handle of the 1st mode of two tennis rackets. 

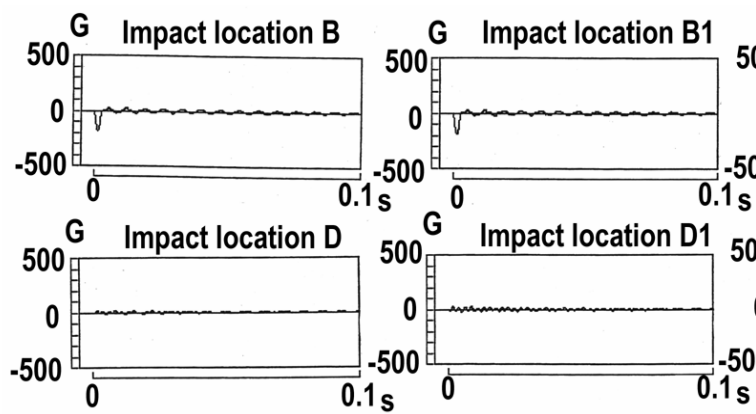

$1 \mathrm{~s} 0$
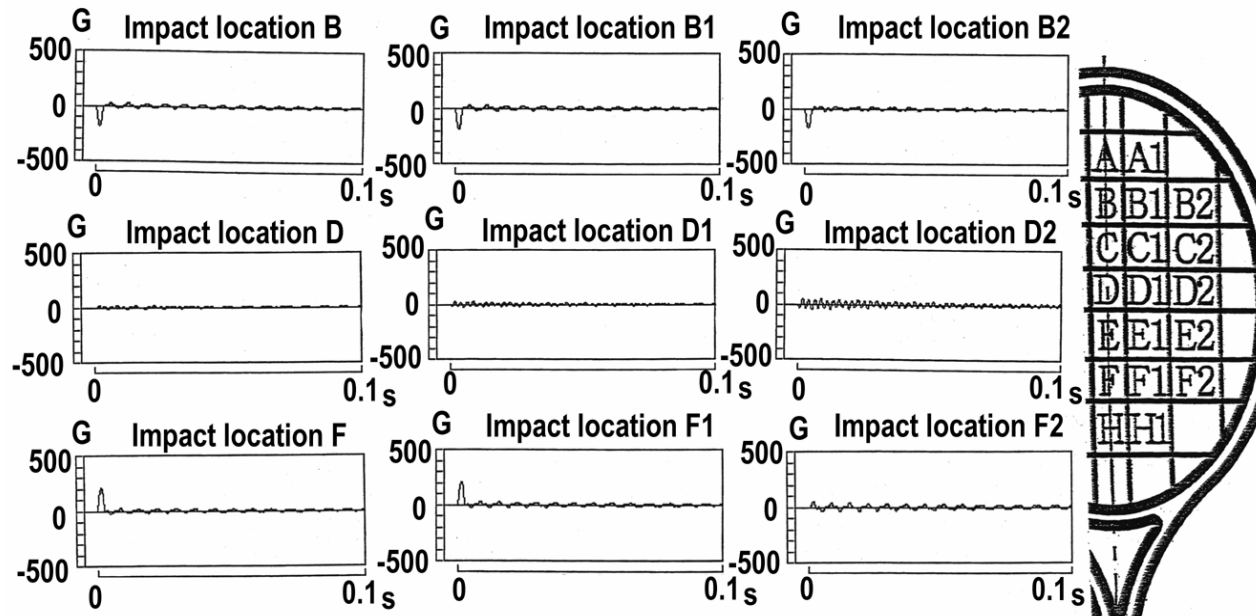

G Impact location F2
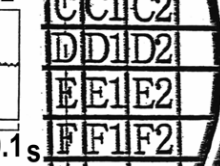

(a) Racket EOS120H: $349 \mathrm{~g}$
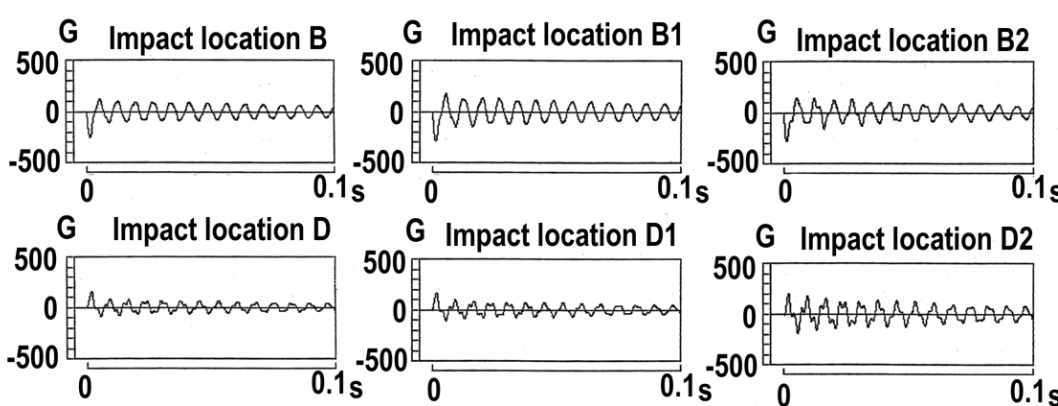

G Impact location D2

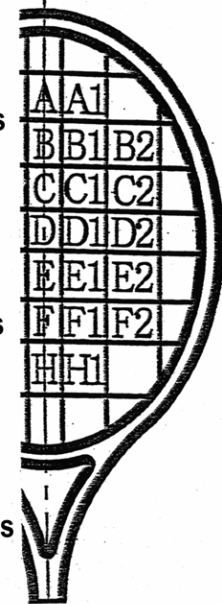

(b) Racket EOS120A: 292 g

Fig.21 Predicted waveform of the shock vibrations at the racket handle on comparing the two freely-suspended rackets with different weight and weight balance when a ball strikes the various locations on the string face along the longitudinal axis and along the short axes at the topside B, center D and nearside F, where the four vibration modes of freely suspended rackets and the shock component due to ball-racket impact are considered. The impact velocity between the ball and the racket is $30 \mathrm{~m} / \mathbf{s}$.

joint on comparing the two rackets with different weight and weight balance when a ball strikes the various locations on the string face along the longitudinal axis and along the short axes at the topside $\mathrm{B}$, center $\mathrm{D}$ and nearside $\mathrm{F}$, where the four vibration modes of hand-held rackets and the shock component due to ball-racket impact are considered. The damping ratio of a hand-held racket in the actual impact is estimated as about 2.5 times that of the one identified by the experimental modal analysis with small vibration amplitude. Furthermore, the damping of the waveform at the wrist joint is estimated as 3 times that at the grip portion of the racket handle. The shock vibrations of super-light racket are much larger than those of conventional weighted and weight balanced racket; the conventional weighted and weight balanced super- large racket is predicted to be very comfortable when the ball is hit with it. 

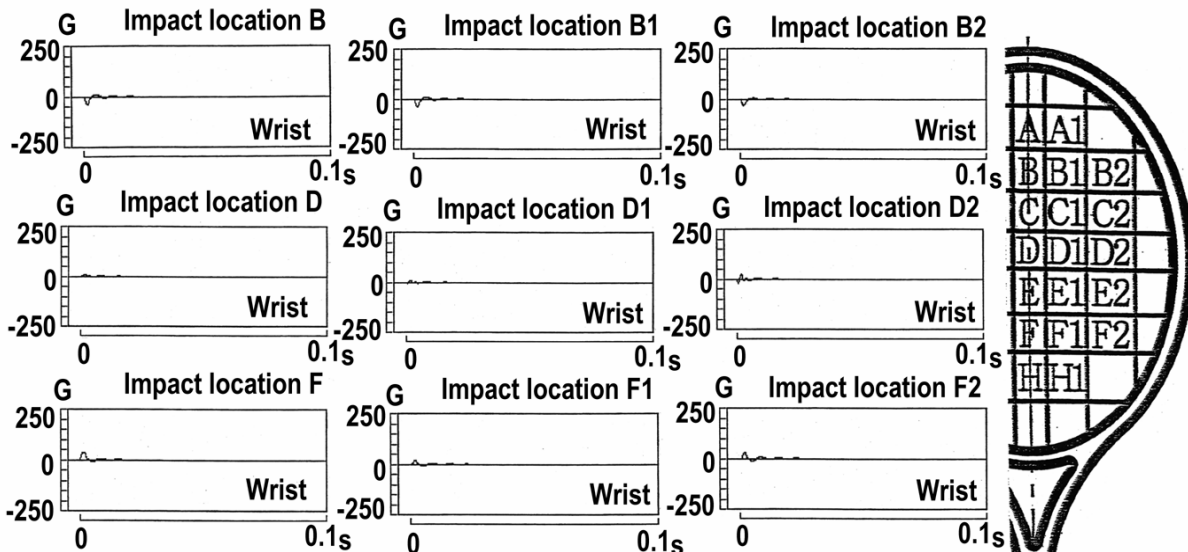

CIC1C2

(a) Racket EOS120H: $349 \mathrm{~g}$
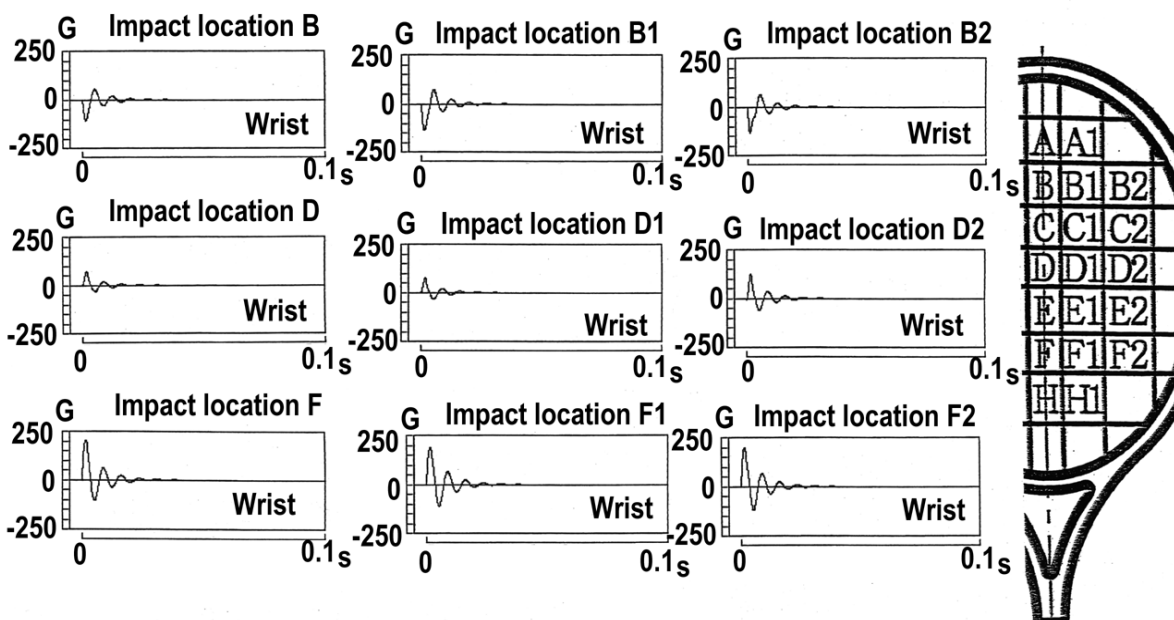

(b) Racket EOS120A:292 g

Fig.22 Predicted waveform of the shock vibrations of the player's wrist joint on comparing the two rackets with different weight and weight balance when a ball strikes the various locations on the string face along the longitudinal axis and along the short axes at the topside B, center D and nearside F, where the four vibration modes of hand-held rackets and the shock component due to ball-racket impact are considered.

\section{Conclusions}

The lightweight racket with handle-light configuration and large head size is recent tendency of high-tech tennis rackets, increasing power or post-impact ball velocity with an increasing racket swing speed. This paper investigated the performance of lightweight tennis racket with super-large head size in terms of feel or comfort. it predicted the effect of the mass and mass distribution of super-large sized rackets on the impact shock vibrations of the racket handle and the player's wrist joint when a player hits flat forehand drive. The prediction is based on the identification of the racket characteristics, the damping of the racket-arm system, equivalent mass of the player's arm system and the approximate nonlinear impact analysis in tennis.

The result showed that the shock vibration of the super-light weight balanced racket with super-large sized head is much larger than that of the conventional weight balanced type racket. It also showed that the sweet area of the former in terms of the shock vibration shifts from the center to the topside on the racket face compared to the latter.

This is because the location of the grip ( $70 \mathrm{~mm}$ from the grip end) on the racket handle is further from the location of the node on the handle of the first mode of super-light racket than that of the conventional weight balanced type racket. The result of the comparison 
showed that the shock vibration of the super-light racket with super-large head size has disadvantage for the shock vibrations at the racket handle and the wrist joint compared to the racket with conventional weight and weight distribution.

\section{Acknowledgements}

The authors are grateful to Dr. Ryoji Tanahashi at Tanahashi Applied Mechanics Labolatory (former chief at Sport Laboratory of Yamaha Company) for his suggestions and encouragement for a long time. Sincere thanks are extended to many students for their help in carrying out this study when senior students at Saitama Institute of Technology.

\section{References}

(1) Casolo,F. \& Ruggieri,G., Dynamic analysis of the ball-racket impact in the game of tennis, Meccanica,Vol.24, (1991), pp.501- 504.

(2) Kawazoe, Y., Tomosue, R., Yoshinari, K. and Casolo, F., Study on the Feel of Tennis Racket at Impact: Mechanism of Impact Shock Vibrations of a Racket Grip and a Player's Wrist Joint, JSME D\&D2000, CD-Rom, (2000), pp.1-6.

(3) Kawazoe,Y., Dynamics and computer aided design of tennis racket. Proc. Int. Sympo. on Advanced Computers for Dynamics and Design'89, (1989), pp.243-248.

(4) Kawazoe,Y., Experimental Identification of Hand-held Tennis Racket Characteristics and Prediction of Rebound Ball Velocity at Impact, Theoretical and Applied Mechanics, Vol.46, (1997), pp.165-176.

(5) Kawazoe,Y., Coefficient of restitution between a ball and a tennis racket, Theoretical and Applied Mechanics, Vol.42, (1993), pp.197-208.

(6) Kawazoe,Y., Impact phenomena between racket and ball during tennis stroke, Theoretical and Applied Mechanics,Vol.41,(1992), pp.3-13.

(7) Kawazoe,Y., Effects of String Pre-tension on Impact between Ball and Racket in Tennis, Theoretical and Applied Mechanics, Vol.43,(1994), pp.223-232.

(8) Kawazoe,Y.,Computer Aided Prediction of the Vibration and Rebound Velocity Characteristics of Tennis Rackets with Various Physical Properties, Science and Racket Sports, E \& FN SPON. (1994), pp.134-139.

(9) Kawazoe, Y., Performance Prediction of Tennis Rackets with Materials of the Wood and the Modern Composites, 5th Japan Int. SAMPE Sympo.\& Exhibition, (1997), pp.1323-1328.

(10) Kawazoe, Y. and Kanda, Y., Analysis of impact phenomena in a tennis ball-racket system (Effects of frame vibrations and optimum racket design), JSME International Journal, Series C, Vol.40, No.1, (1997), pp.9-16.

(11) Kawazoe, Y., Tomosue, R. \& Miura, A., Impact shock vibrations of the wrist and the elbow in the tennis forehand drive: remarks on the measured wave forms considering the racket physical properties, Proc. of Int. Conf. on New Frontiers in Biomechanical Engineering,(1997), pp.285-288.

(12) Kawazoe, Y., TAKEDA, Y. and NAKAGAWA, M., Performance Prediction of Active Piezo Fiber Rackets in Terms of Tennis Power, Journal of System Design and Dynamics, JSME, Vol.4, No.1, (2010), pp.77-90.

(13) Casolo, F., Lorenzi, V. and Sasahara, H., On tennis equipment, impact,simulation and stroke precision, Tennis Science and Technology, Blackwell Science, (2000), pp.83-90. 